\title{
On Approximating Fermion Masses in Terms of Stationary Super-String States
}

\author{
Joseph Towe \\ Department of Physics, The Antelope Valley College, Lancaster, USA \\ Email: jtowe@avc.edu
}

Received January 31, 2013; revised March 3, 2013; accepted March 10, 2013

Copyright (C) 2013 Joseph Towe. This is an open access article distributed under the Creative Commons Attribution License, which permits unrestricted use, distribution, and reproduction in any medium, provided the original work is properly cited.

\begin{abstract}
AdS/CFT correspondence is adopted and fermion masses are modeled as analogues of Weyl curvature states, which occur by hypothesis when closed spin-2 strings sweep out closed world tubes. Admissible curvature states are established by gauge invariance and fundamental mass is attributed to admissible curvature. A consequent spectrum of masses forms an SU(3) symmetry that is invariant under appropriate realizations of the SUGRA GUT interaction. Finally the spin- $\hbar / 2$ nature of the masses that are attributed to curvature emerges as a necessary condition for the relevant SUGRA GUT realizations. Calibration of the proposed model reveals a spectrum of fermion masses that corresponds approximately to observation. Moreover, the proposed model predicts a new quark that is characterized by $I_{3}=-1 / 2$ and by a mass of about $30 \mathrm{GeV} / \mathrm{c}^{2}$.
\end{abstract}

Keywords: String Theory; Conformal Field Theory; Quarks; Super-Gravity

\section{Introduction}

The proposed model is partially motivated by the geometry of Hermann Weyl in which the parallel displacement of a vector around a closed curve admits an increment of vector magnitude. The theory of Weyl associates increments of vector magnitude with curvature states and gauge transformations [1]:

$$
\exp \oint \frac{\mathrm{d} l}{l}=\exp \oint \varphi_{\mu} \mathrm{d} x^{\mu}
$$

The model that is proposed here replaces the parallel transports of vectors around closed curves with the displacements of closed, spin-2 strings that sweep out closed world tubes; i.e. the model that is proposed here replaces Weyl curvature with curvature states that associate with increments of string scale:

$$
W=\oint \frac{\mathrm{d} S}{S},
$$

this analogue of Weyl curvature will be known as $W$-curvature. Based upon a hypothesis introduced by F. London, a curvature state (2) will be regarded as admissible if and only if gauge is preserved in the sense that

$$
\exp [W]=\exp [2 \pi \mathrm{i} n]:
$$

$n= \pm 1$ [2]. An admissible curvature state will be regarded, by hypothesis, as a curvature state that can occur in the physical world; i.e. as an element of a proposed physical geometry.

Secondly, the proposed model considers the compound world tube that is generated when the outer circumference of a closed world tube itself sweeps out a closed world tube:

$$
\int_{0}^{W} \int_{0}^{W} \mathrm{~d} W \mathrm{~d} W=\frac{W^{2}}{2 !} .
$$

The composite (4) is regarded as a second order element of the proposed physical geometry if and only if each of the two world tubes that together constitute the composite (4) preserves gauge in the sense that

$$
\exp \left[W^{n} / n !\right]=\exp [2 \pi \mathrm{i} n]:
$$

$n= \pm 1, \pm 2$. Finally it is argued that generalizations of the proposed second order process generate composites:

$$
\int_{0}^{W} \cdots \int_{0}^{W} \mathrm{~d} W \cdots \mathrm{d} W=\frac{W^{n}}{n !}
$$

or

$$
W-\text { curvature }=\left[W^{n} / n !\right]:
$$

$n= \pm 1, \pm 2, \pm 3, \cdots, \pm m$. Once again, each curvature state (7) is regarded as an element of the proposed physical 
geometry if and only if

$$
\exp \left[W^{n} / n !\right]=\exp [2 \pi \mathrm{i} n]:
$$

$W>0, n= \pm 1, \pm 2, \cdots, \pm m$ [3].

AdS/CFT correspondence and the string background $\mathrm{AdS}_{7} \mathrm{XS}^{4}$ are now adopted by hypothesis $[4,5]$.

\section{Theoretical Lepto-Quark States}

The $W$-curvature classes (7) (established as admissible by (8)) will now be associated with specific masses, which are regarded as equivalent to the $\mathrm{W}$-curvature states that are described by (7) (the proposed model parallels Wheeler's ideal, which attributes mass to curvature [6]). These masses are initially regarded as significant because they are attributed to curvature, but they assume additional importance in the context of the proposed model because they establish a context in which spin- $(\hbar / 2)$ is intrinsically associated with postulated orientation states $A_{L}$ and $a_{L}$ as a necessary condition for a relevant realization of SUGRA GUT interactions. Specifically an admissible, closed world tube (or a closed, compound world tube) is interpreted, by hypothesis, as a transformation from the mass-less, spin- $2 \hbar$ field, which will be designated $\psi_{L}$, to a massive composite $a_{L} \psi_{L} \bar{A}_{R}$, where the pairs $A_{L}, \bar{A}_{R}$ and $a_{L}, \bar{a}_{R}$ consist of CPT conjugates. By hypothesis, these four fields share a common spin magnitude. Each composite $a_{L} \psi_{L} \bar{A}_{R}$ is therefore characterized by a net spin magnitude of $2 \hbar$. Finally it is observed that $A_{L}, \bar{A}_{R}$, $a_{L}$ and $\bar{a}_{R}$ can interact super-gravitationally: provided that composite triplets $a_{L} a_{L} a_{L}, a_{L} a_{L} A_{L}, A_{L} A_{L} a_{L}$ and

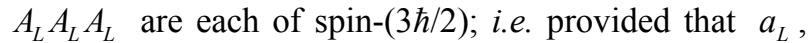
$\bar{a}_{R}, A_{L}$ and $\bar{A}_{R}$ are individually characterized by a spin of $\hbar / 2$; and provided that the above described triplets are characterized by a second quantum number that ranges over three values and that the consequent triplets $a_{L}^{(1)} a_{L}^{(2)} a_{L}^{(3)}, a_{L}^{(1)} a_{L}^{(2)} A_{L}^{(3)}$ etc., each represent sufficient diversity in this new quantum number to avoid violation of the Pauli exclusion principle. Subsequently the quantum numbers "(1)", "(2)" and "(3)" will be regarded as referring to "generation". It should be noted that if the SUGRA GUT interaction depicted by Figure 1 is to occur (in the relevant forms which preserve the Figure 2 symmetry), then it is necessary that the constituents $a_{L}$ and $\bar{A}_{R}$ of the generic composite $a_{L} \psi_{L} \bar{A}_{R}$ associate with a single generation. In the context of this model, the constituents $a_{L}$ and $\bar{A}_{R}$ are respectively interpreted as a lepton and an anti-quark of the same generation.

\section{Calibration}

The postulated model is calibrated by identification of the $W$-curvature class $W^{6}$ with the most massive leptoquark state that is exclusively characterized by $I_{3}=+1 / 2$. Specifically, $W^{6} / 6$ ! is assigned the value $\left[180 \mathrm{GeV} / \mathrm{c}^{2}\right] / 6$ !

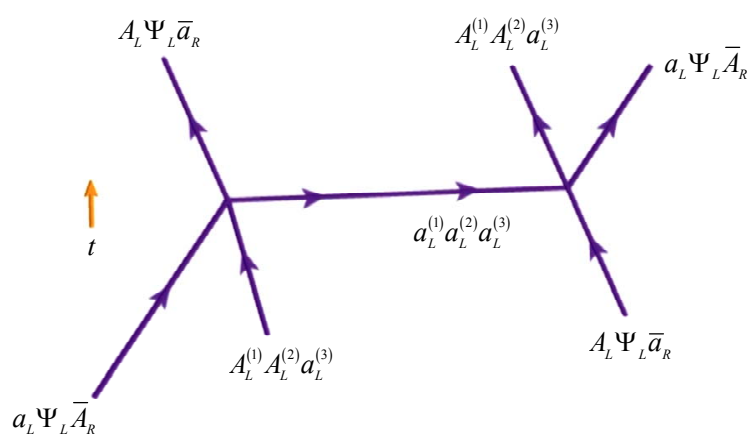

Figure 1. Pure SUGRA interaction involving postu-lated generic composites.

$=0.25 \mathrm{GeV} / \mathrm{c}^{2}$, so that

$$
W^{6}=6 !(0.25) \mathrm{GeV} / \mathrm{c}^{2}=180 \mathrm{GeV} / \mathrm{c}^{2},
$$

where $W^{6}$ is assigned to the quark-lepton state $A_{L}^{(V I)} \Psi_{L} \bar{a}_{R}^{(V I)}=T_{L} \psi_{L} v_{R}^{\tau^{+}}$and where $180 \mathrm{GeV} / \mathrm{c}^{2}$ is beliveed to be the approximate mass of the top quark (Since the mass of the tauon's neutrino is relatively negligible, the mass (9) is also regarded as approximating that of the heaviest lepto-quark state that is characterized by $I_{3}=$ $+1 / 2$ ). The Roman numerals I through VI in Equation (9) through (14) refer to six states that will subsequently be identified as "six generations of $I_{3}$ conjugates".

The following equations are equivalent to (9) and collectively indicate a spectrum of masses:

$$
\begin{aligned}
& W^{5}=A_{L}^{(V)} \Psi_{L} \bar{a}_{R}^{(V)}=5 !(0.25) \mathrm{GeV} / \mathrm{c}^{2}=30 \mathrm{GeV} / \mathrm{c}^{2}, \\
& W^{4}=A_{L}^{(I V)} \Psi_{L} \bar{a}_{R}^{(I V)}=4 !(0.25) \mathrm{GeV} / \mathrm{c}^{2}=6 \mathrm{GeV} / \mathrm{c}^{2}, \\
& W^{3}=A_{L}^{(I I)} \Psi_{L} \bar{a}_{R}^{(I I I)}=3 !(0.25) \mathrm{GeV} / \mathrm{c}^{2}=1.5 \mathrm{GeV} / \mathrm{c}^{2}, \\
& W^{2}=A_{L}^{(I I)} \Psi_{L} \bar{a}_{R}^{(I I)}=2 !(0.25) \mathrm{GeV} / \mathrm{c}^{2}=0.5 \mathrm{GeV} / \mathrm{c}^{2},
\end{aligned}
$$

and

$$
W=W A_{L}^{(I)} \Psi_{L} \bar{a}_{R}^{(I)}=0.25 \mathrm{GeV} / \mathrm{c}^{2},
$$

Interpretation of the mass (10) will be deferred until the massive states described by expressions (11) through (14) have been interpreted. The theoretical mass represented by (11) motivates the association of (11) with the spin- $2 \hbar$ composite $B_{L} \psi_{L} \tau_{R}^{+}$or $\tau_{L}^{-} \psi_{L} \bar{B}_{R}$, where $B_{L}$ is an LH bottom quark, where $\bar{B}_{R}$ is an RH anti-bottom quark (a mass of about $4.3 \mathrm{GeV} / \mathrm{c}^{2}$ ), where $\tau_{L}^{-}$is an $\mathrm{LH}$ tauon (a mass of about $1.7 \mathrm{GeV} / \mathrm{c}^{2}$ ) and where $\tau_{R}^{+}$is an $\mathrm{RH}$ anti-tauon. The bottom quark and the tauon are regarded as $I_{3}=-1 / 2$ partners in the heavy generation.

The mass of expression (12) motivates the association of (12) with the composite spin-2 $2 \hbar$ field $C_{L} \psi_{L} v_{R}^{\mu^{+}}$or $v_{L}^{\mu^{-}} \psi_{L} \bar{C}_{R}$, where $C_{L}$ and $\bar{C}_{R}$ respectively represent the LH charmed quark and the RH anti-charmed; and where $v_{L}^{\mu^{-}}$and $v_{R}^{\mu^{+}}$respectively represent the LH muon's neutrino and the RH anti-muon's neutrino. The charmed 
quark and the muon's neutrino are regarded as $I_{3}=+1 / 2$ partners in the moderately heavy generation.

The mass of expression (13) motivates the association of (13) with the composite, spin-2 $\hbar$ field $e_{L}^{+} \psi_{L} S_{R}$ or $\bar{S}_{L} \psi_{L} e_{R}^{-}$, where $S_{R}$ and $\bar{S}_{L}$ respectively represent the RH strange quark and the LH anti-strange, and where $e_{R}^{-}$ and $e_{L}^{+}$respectively represent the $\mathrm{RH}$ electron and the $\mathrm{LH}$ anti-electron. The strange quark and the right-handed electron are (in the proposed model) regarded as $I_{3}$ partners in the light generation.

The mass of expression (14) motivates the association of (14) with the average mass of the two spin- $2 \hbar$ states $U_{L} \psi_{L} v_{R}^{e^{+}}$and $D_{L} \psi_{L} e_{R}^{+}$, where $U_{L}$ and $D_{L}$ respectively represent the LH up quark and LH down quark, where $\psi_{L}$ represents a mass-less $\mathrm{LH}$ spin- $2 \hbar$ field and where $v_{L}^{e^{-}}$and $e_{L}^{-}$respectively represent the LH electron's neutrino and the LH electron. The up quark and the LH electron's neutrino are regarded as $I_{3}=+1 / 2$ partners in the light generation, and the down quark and LH electron are regarded as $I_{3}=-1 / 2$ partners in the light generation. Note that the masses described by expressions (11), (12), (13) and (14) are approximately equal to those determined by observation $[7,8]$.

To interpret the mass that is described by expression (10), it is first observed that the LH muon $\mu_{L}^{-}$is not included in the earlier discussion. Accordingly, the mass that is described by (10) is interpreted as the spin- $2 \hbar$ composite $7_{L} \psi_{L} \mu_{R}^{+}$or $\mu_{L}^{-} \psi_{L} \overline{7}_{R}$. Paralleling the earlier discussion, the composites $7_{L} \psi_{L} \mu_{R}^{+}$and $\mu_{L} \psi_{L} \overline{7}_{R}$ are interpreted as lepto-quark states (which consist of conjugates that form composites with spin- $2 \hbar$ fields $\psi_{L}$ ), the constituents of which share an $I_{3}$ classification and a generational classification. Thus the $7_{L}$ is interpreted as an unobserved LH quark that is characterized by $I_{3}=$ $-1 / 2$ and is regarded as an element of the moderately heavy generation. Finally, since the mass of the $\mu_{L}^{-}$is relatively negligible, the mass of the newly predicted quark that is associated with expression (10) will be designated as approximately $30 \mathrm{GeV} / \mathrm{c}^{2}$.

The spin- $2 \hbar$ composites (9) through (14) are associated with three generations of $I_{3}$ generators. These elements are therefore interpreted as constituting an SU(3) symmetry, the preservation of which is regarded as equivalent to the conservation of $I_{3}$ and generation:

Referring to Figure 2, the counterclockwise sequence beginning at $180 \mathrm{GeV} / \mathrm{c}^{2}$ indicates, in order of occurrence, the masses that are described by expressions (9) through (14). Again the mass $0.25 \mathrm{GeV} / \mathrm{c}^{2}$ and the associated $I_{3}$ value of $I_{3}=0$ represent the averages of the masses and $I_{3}$ numbers of the up quark (together with its $I_{3}$ lepton partner) and the down quark (together with its $I_{3}$ lepton partner).

Note that the Figure 2 symmetry is partially broken because the vertices represent a variety of masses. Note however that an appropriate realization of the SUGRA

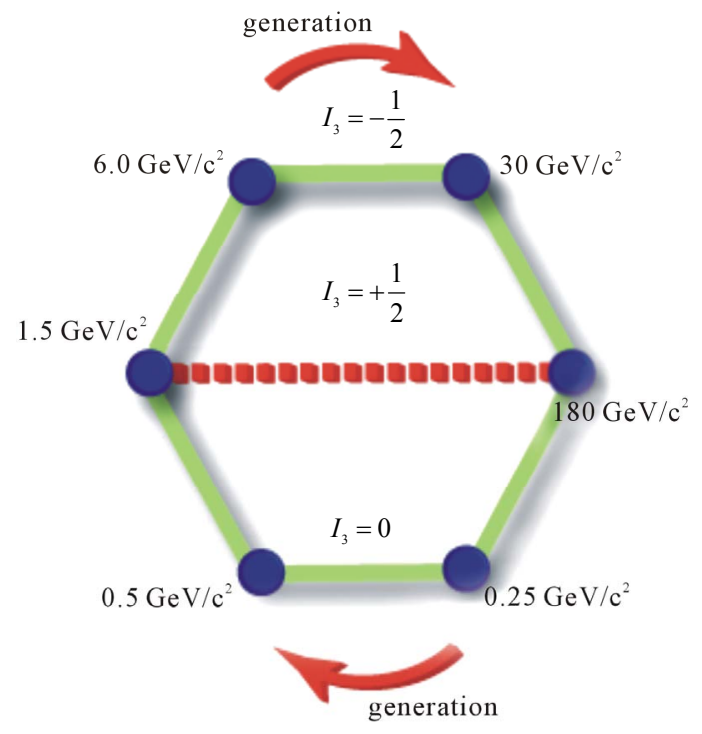

Figure 2. World symmetry.

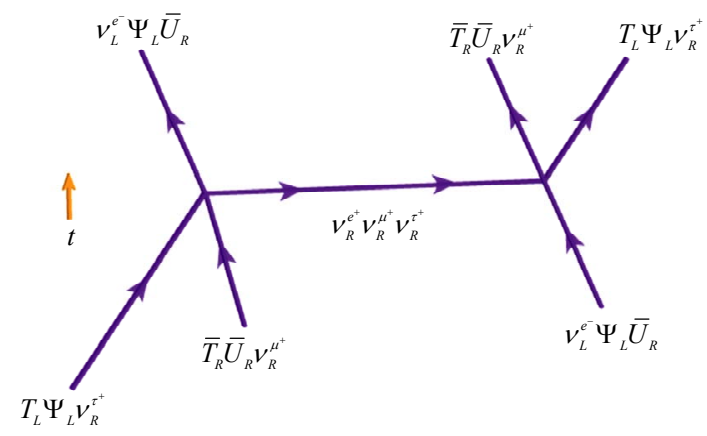

Figure 3. A pure SUGRA GUT interaction.

GUT interaction can preserve the partially broken symmetry that is depicted in Figure 2 by beginning and terminating at a single vertex. Specifically, the Figure 2 symmetry is preserved by the SUGRA GUT interaction.

Note that preservation of the Figure 2 symmetry by the Figure 3 interaction is indeed equivalent to the conservation of $I_{3}$ and generation by the Figure 3 interaction.

\section{Conclusion}

The proposed model is geometrically characterized by Ricci flatness (all curvature is identified with the analogues (7) of Weyl curvature) and by the SU(3) symmetry that is described by Figure 2. This model appears to represent an increment of progress because it is characterized by a uniqueness that is established by gauge invariance and because significant physical consequences and predictions emerge.

\section{Acknowledgements}

Figures designed by R. James Towe. 


\section{REFERENCES}

[1] H. Weyl, "Space, Time and Matter," Metheun and Co. Ltd., London, 1922.

[2] F. London, "Quantenmechanische Deutung der Theorie von Weyl," Zeitschrift fur Physik, Vol. 42, No. 5-6, 1927, pp. 375-389. doi:10.1007/BF01397316

[3] J. Towe, "Stationary String States as Theoretical Fermions," Advanced Studies in Theoretical Physics, Vol. 6, No. 8, 2012, pp. 369-377.

[4] O. Aharony, S. Gubser, J. Maldacina, H. Ooguri and Y. Oz, "Large N-Theories, String Theory and Gravity," 1999, in press.
[5] E. D'Hoker and D. Freedman, "Super-Symmetric Gauge Theories and the AdS/CFT Correspon-Dance," 2002, in press.

[6] J. A. Wheeler, "Curved, Empty Space as the Building Material of the Physical World," In: E. Nagel, Ed., Logic, Methodology and the Philosophy of Science, Stanford University Press, Palo Alto, 1962.

[7] R. Bock and A. Vasilesca, "Particle Detector Brief Book," 1999.

[8] SLAC Virtual Visitor Center, "SLAC National Accelerator Laboratory," Menlo Park, 2009. 